\title{
BMJ Open Systematic review of patient- engagement interventions: potentials for enhancing person-centred care for older patients with multimorbidity
}

\author{
Mathilde Bendix Søgaard (D) , Katrine Andresen (D), Maria Kristiansen
}

To cite: Søgaard MB, Andresen K, Kristiansen M. Systematic review of patientengagement interventions: potentials for enhancing personcentred care for older patients with multimorbidity. BMJ Open 2021;11:e048558. doi:10.1136/ bmjopen-2020-048558

- Prepublication history and additional supplemental material for this paper are available online. To view these files, please visit the journal online (http://dx.doi.org/10.1136/ bmjopen-2020-048558).

Received 05 January 2021 Accepted 22 November 2021

Check for updates

(c) Author(s) (or their employer(s)) 2021. Re-use permitted under CC BY-NC. No commercial re-use. See rights and permissions. Published by BMJ.

Department of Public Health \& Center for Healthy Aging, University of Copenhagen, Copenhagen, Denmark

Correspondence to Dr Maria Kristiansen; makk@sund.ku.dk

\section{ABSTRACT}

Introduction Person-centred care based on systematic and comprehensive patient-engagement is gaining momentum across healthcare systems. Providing care that is responsive to the needs, values and priorities of each patient is important for patients, relatives and providers alike, not least for the growing population of older patients living with multi-morbidity and associated complex care trajectories.

Objectives The aim of this systematic review is to investigate the effects of patient engagement interventions for older patients with multimorbidity.

Methods Systematic review conducted in August 2021. Two reviewers independently screened the international databases Embase and PubMed. Reviewers carried out duplicate and independent data extraction and assessment of study quality. Grading of Recommendations Assessment, Development and Evaluation was used to assess the quality of the evidence for each study.

Results We included twelve studies from primary care setting and hospitals. The included studies were heterogeneous in terms of characteristics of populations, types of interventions to enhance patient-engagement, outcome measures and length of follow-up. Nine of the 12 included studies found significant improvements in health and patient-reported outcomes such as higher qualityadjusted life-years, fewer hospital visits and disease specific symptoms. Quality of the included studies was of low to moderate.

Conclusion This review identifies potential beneficial effects of interventions to enhance patient-engagement in older adults with multimorbidity. Nevertheless, the limited and very diverse evidence-based calls for more robust studies into efficient approaches to engaging older adults with multimorbidity in care trajectories.

\section{INTRODUCTION}

Person-centred care is defined as care that is based on elicitation of and responsiveness to the individual patient's needs, values, resources and life situation. ${ }^{1}$ In increasingly complex and prolonged treatment trajectories, engaging older patients in a timely, systematic and holistic manner may improve experiences and outcomes for patients and relatives, and enhance meaningfulness among

\section{Strengths and limitations of this study}

- This systematic review explores the understudied field of interventions to enhance patientengagement in the growing population of older adults with multimorbidity.

- Included studies are not limited to specific healthreported and patient-reported outcomes in order to capture the broad effectiveness of these interventions.

- Meta-analysis was not possible due to heterogeneity in methods and outcomes.

- Quality of the included studies was low to moderate overall, hence there is a need for more robust studies using a range of outcomes to identify best practices in patient-engagement. in the context of multimorbidity in old age.

healthcare professionals. Furthermore, it may support adequate use of scarce healthcare resources as treatment plans are tailored to individual needs, potentially improving engagement of more disadvantaged patients and ultimately decreasing social inequality in healthcare utilisation. ${ }^{23}$

Identifying the best care trajectory in the light of the uniqueness of each patient's circumstances is particularly important for the growing population of older patients living with multimorbidity, defined as patients living with two or more coexisting long term conditions, ${ }^{4}$ and associated polypharmacy requiring prolonged and complex care trajectories across care settings. ${ }^{5-7}$ In the context of population ageing and increased multi-morbidity, more systematically and timely offered conversations with patients related to future scenarios and priorities is crucial. This includes a range of complex decisions on prognosis, treatment options and prioritising care at the end of life driven by patient perspectives on what is acceptable and meaningful to him/her. Person-centred care requires empowered patients who are met by a 
responsive and accessible healthcare system with a culture of engagement, sufficient time, and a skill-set that nurtures daily practices based on unlocking patient perspectives and delivering coherent care to reflect these. ${ }^{389}$ While there is overall agreement concerning the importance of person-centred care, there is little scientific evidence documenting significant outcomes of patient engagement regarding patient satisfaction, enhanced shared decision-making (SDM), adjustment of treatment plans, or use of resources. ${ }^{10-14} \mathrm{~A}$ recent systematic review ${ }^{15}$ aimed at assessing the effect of interventions for older patients with multimorbidity aiming to involve them in decision-making in primary care consultations found too limited evidence to interpret with certainty. This systematic review included only randomised controlled trial's (RCTs) in primary healthcare. To investigate this topic further, we included both RCTs and non-randomised studies in primary and secondary healthcare settings. More focus on patient-engagement tools as interventions to enhance person-centred care in clinical encounters is needed to provide a more substantive evidence base to guide prioritisation and implementation into mainstream healthcare delivery. ${ }^{16}$ The aim of the systematic review is to investigate the current evidence for effectiveness of patient-engagement tools in enhancing person-centred care for older adults $(60+$ year) with more than one disease.

\section{METHODS}

\section{Literature search and study selection}

The review is based on systematic literature searches conducted in December 2019 and updated August 2021 using the databases PubMed and Embase. Furthermore, reference lists of included articles were assessed to identify additional peer-reviewed articles. The complete list of search terms, including Medical Subject Headings $(\mathrm{MeSH})$ terms and free text terms, is presented in online supplemental appendix 1.

The software DistillerSR was used to screen and review the studies. Data were independently extracted onto a customised data extraction sheet in DistillerSR by two reviewers, and any discrepancies were resolved by discussion.

The eligibility criteria for study inclusion were as follows:

- Population: Older adults above the age of 60 living with two or more coexisting diseases.

- Intervention: Patient engagement intervention in healthcare system settings.

- Comparison groups: Older patients who received usual care.

- Outcome: Any patient-related outcome, for example, reduced symptoms of disease, reduced duration of disease, reduced costs and reduced hospital stay or rehospitalisation.

We included quantitative observational studies such as prospective and retrospective cohort studies and RCTs. Studies in any geographical area in healthcare systems, thus encompassing both primary and secondary care settings, were included, and only studies written in English and in one of the Scandinavian languages (Danish, Swedish or Norwegian) were included.

Two investigators independently screened the titles, abstracts and full texts for inclusion and exclusion criteria. We excluded commentaries, editorials and studies that did not directly apply a patient-engagement intervention as an exposure. We did not exclude studies based on publication date.

First, the titles of the 805 studies were screened for eligibility. Second, duplicates were removed. Third, the abstracts of the studies were screened. Fourth, the full texts of studies initially assessed as relevant for the review were checked against our inclusion or exclusion criteria. Disagreements between the two investigators were resolved by consensus. Figure 1 shows reasons for exclusion for potentially eligible studies.

\section{Data extraction}

Two investigators independently extracted information on characteristics of participants, study design, patient engagement intervention and outcomes. Discrepancies in data extraction were resolved by consensus between the two investigators.

\section{Quality assessment}

The included studies encompassed a combination of RCT and observational studies. To assess the quality of evidence, we used Grading of Recommendations, Assessment, Development and Evaluation (GRADE) for all the included studies. GRADE is a transparency framework and is the most widely adopted tool for grading the quality of evidence. The checklist assesses quality of the study across eight domains. The evidence level can be rated down or up depending on missing or existing domains. GRADE certainty ratings have four levels as follows: very low, low, moderate, high. Very low means that the true effect is probably markedly different from the estimated effect. High means that the authors have a lot of confidence that the true effect of is similar to the estimated effect. ${ }^{17}$ To assess the domain risk of bias within GRADE we used two different measures depending on whether the study was randomised or non-randomised. In non-randomised studies we used ROBINS-1 to assess risk of bias, which is a tool to understand and appraise strengths and weaknesses in non-randomised studies. ${ }^{18}$ In RCTs, we used Cochrane Collaboration's tool for assessing risk of bias. ${ }^{19}$ Two investigators independently performed a quality assessment of each study. Disagreements were resolved by consensus.

\section{Patient and public involvement statement}

Patients were not involved in this systematic review.

\section{RESULTS}

\section{Description of included studies}

Figure 1 shows the Preferred Reporting Items for Systematic Reviews and Meta-Analyses diagram in the phases from the 805 studies that were identified to the 12 studies 


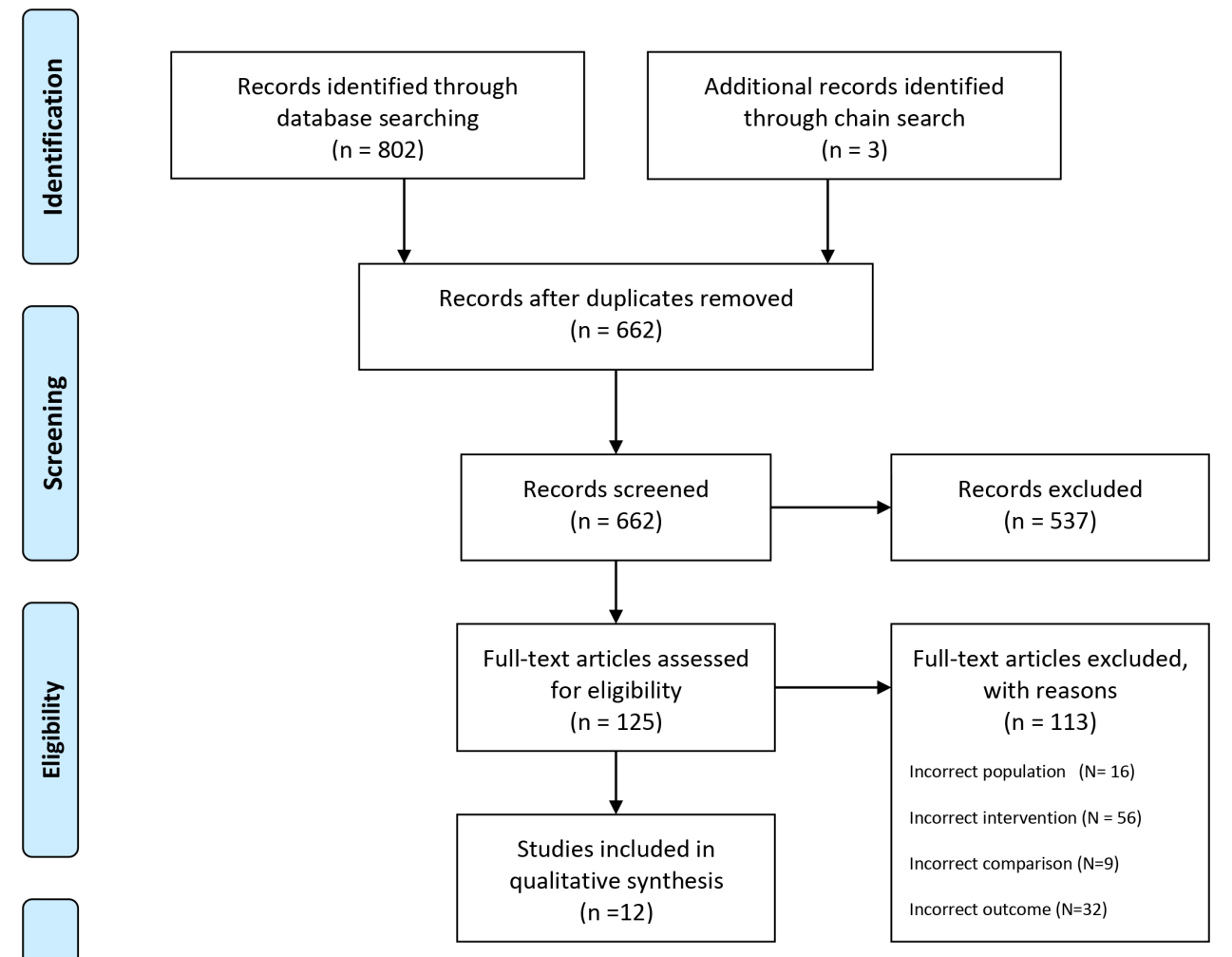

Figure 1 Flow chart.

that met the inclusion criteria. The main reason for exclusion were due to lack of specific interventions. Table 1 depicts the characteristics of the twelve included studies. The included studies were mainly RCT studies.

The sample sizes of the twelve included studies ranged widely across studies (mean: 684 patients, range 79-2282). Five studies (42\%) were conducted in the Europe, four $(33 \%)$ in USA and three $(25 \%)$ elsewhere. The most common study design was RCT $(n=8 ; 67 \%)$, followed by Cohort/observational $(\mathrm{n}=3 ; 25 \%)$ and quasi-experimental design $(\mathrm{n}=1 ; 8 \%)$. Most studies were conducted in a primary care setting $(\mathrm{n}=7 ; 58 \%)$. A total of five studies $(42 \%)$ were conducted in general hospitals involving patients from a range of specialties. In the studies, the participants have many different diseases such as diabetes, chronic heart failure or dementia. The mean age in the studies was +60 years with a range: $60-84$ years, however, there were two studies that did not indicate the mean age but included an $18+$ year population with most $60+$ years. A wide range of multimorbidities is represented in the included studies with some focusing more widely on engagement of patients with multiple coexisting diseases whereas others targeted specific diseases in patients with comorbidities.

The included studies used different patient engagement interventions, such as coaching, healthcare communication, goal setting interventions, self-management programme, 3D intervention, prompt list, and disease-specific sessions. Different types of primary outcomes were used in studies such as Patient Activations Measurements (PAM), self-rated health (SRH), hospitalisation, use of health services, change in clinical outcomes (eg, body mass index, blood pressure (BP), blood glucose), Modified Barthel Index (MBI) and quality of life. Disease specific outcomes such as BP, cholesterol level and blood glucose were used in some of the studies.

\section{Quality of included studies}

Figure 2 shows the quality assessment for each included study. Three studies were assessed to be of high quality in all domains apart from one, which was judged to be low or moderate. An additional five studies were assessed to be of high quality apart from two domains, which was judged to be low or moderate. The last four studies were only judged to be of high quality in one or none of the five domains.

Risk of bias for RCTs is shown in figure 3. All studies had high risk of bias due to blinding of participants and personnel (performance bias). Furthermore, four studies were rated to high risk of blinding of outcome assessment (detection bias). ${ }^{20-23}$ 


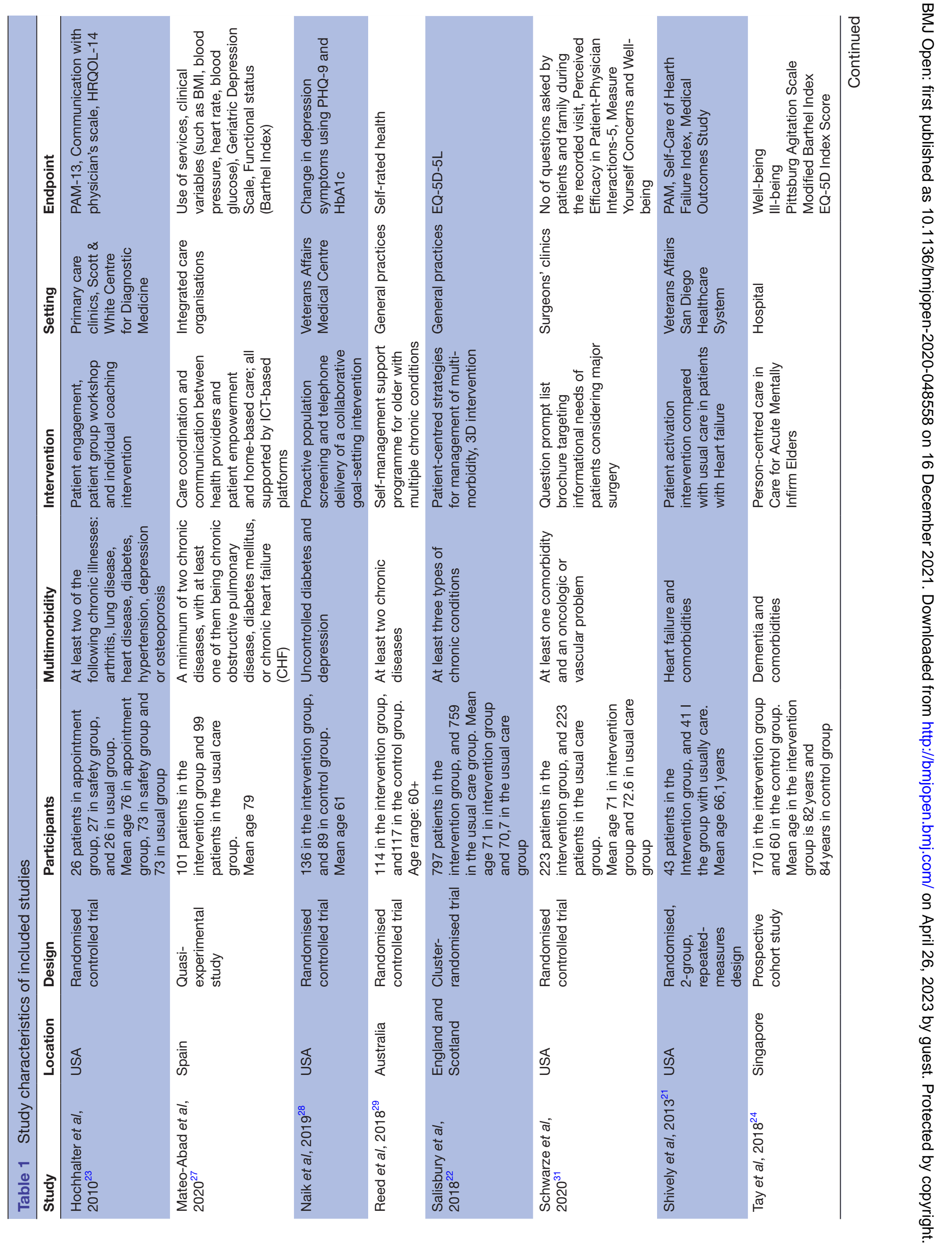




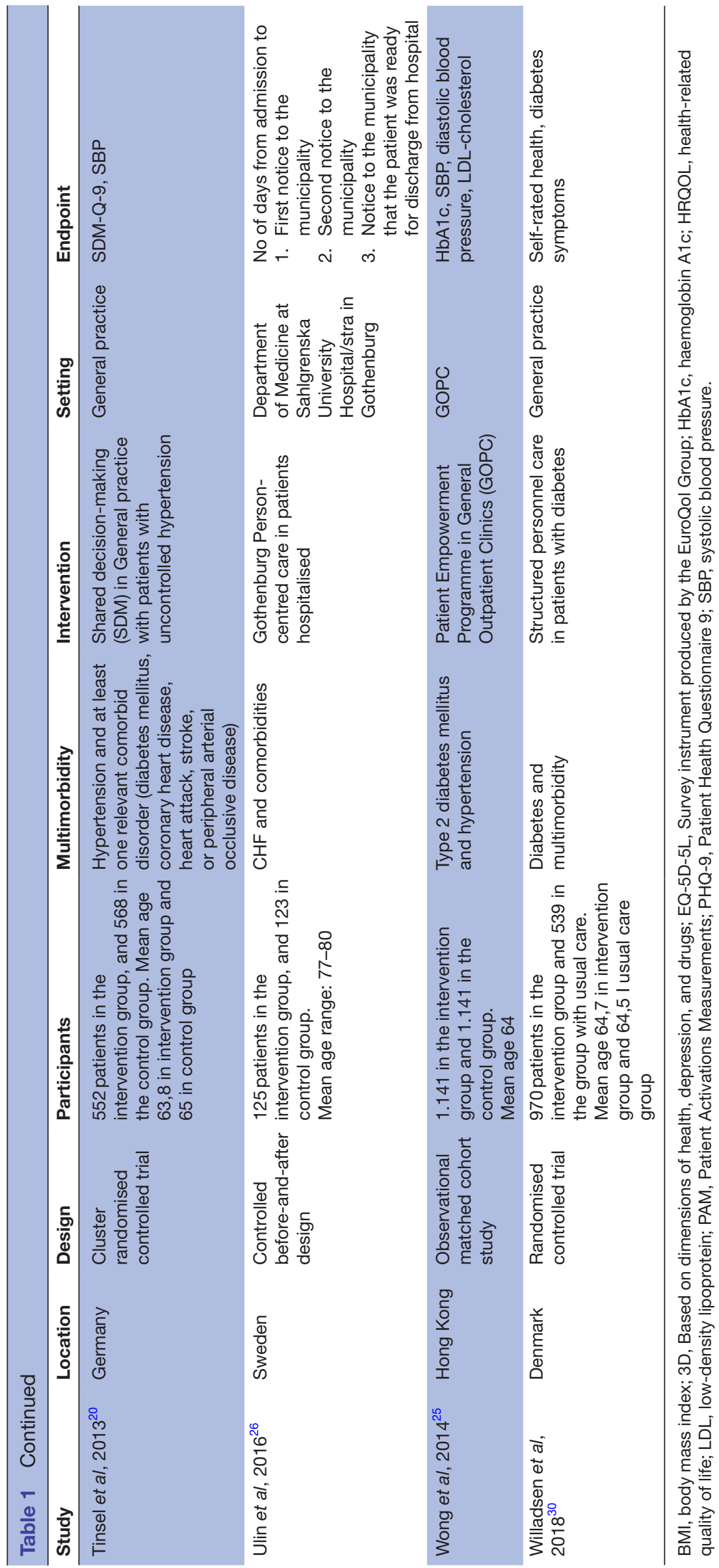



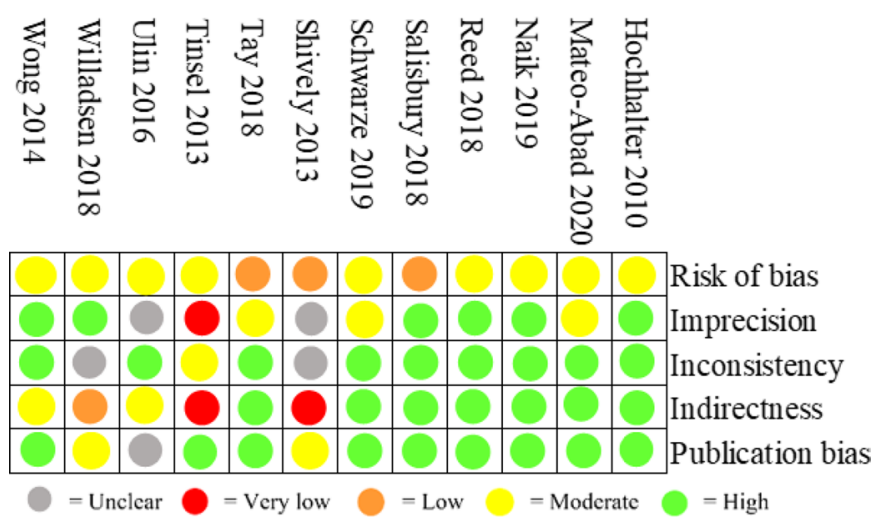

Figure 2 Total GRADE. GRADE, Grading of

Recommendations Assessment, Development and

Evaluation.

Figure 4 shows risk of bias for non-randomised studies. Two studies were rated to have high risk of bias, and two studies were rated moderate risk of bias. One study had high risk of bias due to confounding, ${ }^{24}$ and another study had high risk of bias due to deviations from intended intervention. ${ }^{25}$ One study rated to moderate risk of bias had moderate risk of bias in five categories,${ }^{26}$ and the other had moderate risk of bias in two categories. ${ }^{27}$ All four studies had unclear risk of bias in more than one category.

In total, the quality of the included studies was of low to moderate and some aspects of quality assessment and risk of bias were unclear across the included studies.

\section{Effect of the interventions}

The included studies are using different endpoints to measure the effect of patient engagement interventions. Nine of the included studies found significant effect of interventions.

An RCT by Naik et $a l^{28}$ measure the effect of the intervention Healthy Outcomes Through Patient Empowerment (HOPE) which is a 6 months goal-setting intervention targeting depression symptoms and diabetes self-care through nine telephone-delivered coaching sessions. The HOPE intervention used an electronic data warehouse to identify specific high-risk population, followed by telephone screening and training of clinicians to deliver a structured telehealth intervention. The endpoints in

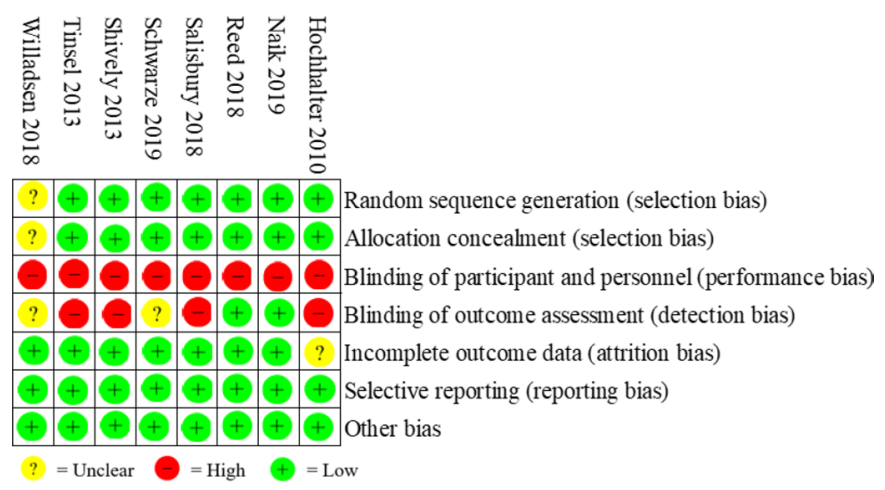

Figure 3 Risk of bias for randomised studies.

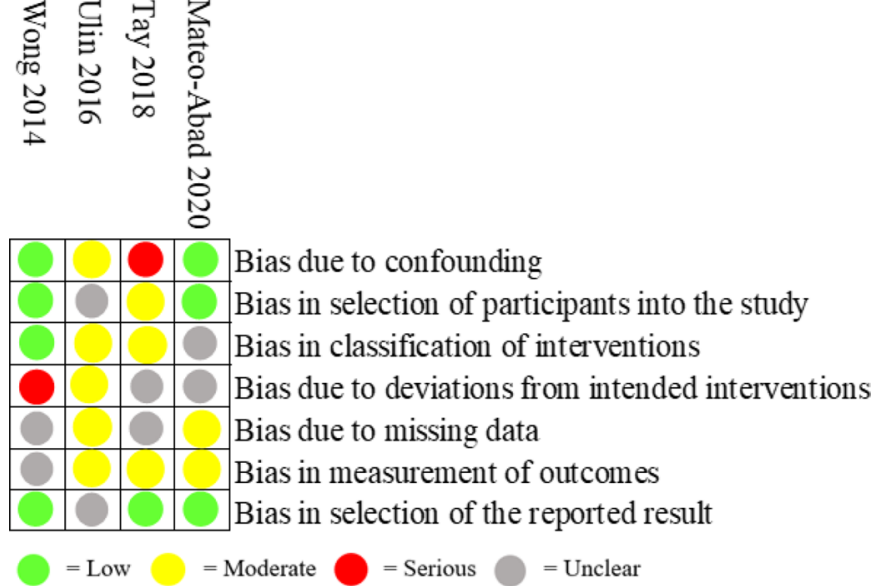

Figure 4 Risk of bias for non-randomised studies.

this study was depression symptoms with The Patient Health Questionnaire (PHQ)-9 and glycaemic control with haemoglobin A1c (HbA1c). They did not find any improvement in 6-month follow-up, but found improvement in PHQ-9 after 12-month follow-up; HOPE (mean (SD) baseline: 15.8 (4.2) to 6 months: 10.9 (6.1) and 12 months to 10.1 (6.5)) compared with enhanced usual care (EUC) (mean (SD) baseline 16.2 (4.0) to 6 months $12.4(6.0)$ ) and 12 months to 12.6 (6.0). The PHQ-9 differences between HOPE and EUC were statistically significant at 6 months (mean diff., $1.74 ; 95 \%$ CI 0.14 to 3.33; $\mathrm{p}=0.03$ ) and 12 months (mean diff., 2.14; $95 \% \mathrm{CI}$ 0.18 to $4.10 ; \mathrm{p}=0.03$ ).

The study by Reed $e t a l^{29}$ examined the effect of the intervention Chronic Disease Self-Management Support (CDSMS) which is a set of tools (Partners in Health scale, Cue and Response interview, Problems and Goals assessment) and a structured process that enable clinicians and patients to collaboratively assess self-management behaviour, identify problems, set goals and develop individual care plans that address key self-care, medical, psychosocial and care problems. Participants in each programme received three home visits and four follow-up phone calls over a 6 -month period from a clinician. The population were recruited from five general practices in Adelaide, Australia. The study used SRH as endpoint, and they with an intention-to-treat analysis that CDSMS participants were more likely than control participants to report improved SRH at 6 months (R, 2.50; 95\% CI 1.13 to $5.50 ; \mathrm{p}=0.02$ ).

An RCT by Shively et $a t^{21}$ implemented a 6 -month programme developed to enhance self-management in older heart failure patients. The programme consisted of individualised goal setting according to baseline activation level. The interventions population was invited to participate through a follow-up visit at the Veterans Affairs San Diego Healthcare system. The study used PAM, Self-Care of Heart Failure Index, Medical Outcomes Study and hospital visits to measure the effect of the intervention. The intervention showed improvement in PAM-score, the intervention group compared with the usual care group 
showed a significant increase in PAM-scores from baseline to 6 months (significant group by time interaction, $\mathrm{F}=3.73, \mathrm{p}=0.03$ ) and fewer hospital visits compared with usual group.

A prospective naturalistic cohort study by Tay et $a t^{24}$ examined the effect of the intervention Care for Acute Mentally Infirm Elders (CAMIE) which adopt a personcentred care protocol with specialised psychosocial interventions, minimally obtrusive medical care and physical restraints-free practice targeting patients with dementia. The study population were recruited at a hospital, and all patients received standard treatment. Patients were admitted to the CAMIE unit if they suffered from confusion due to dementia, with/without delirium based on the confusion assessment method criteria, and concomitant acute medical problems. The study used MBI function and well-being and European Quality of Life (EuroQol) to measure the CAMIE intervention. CAMIE patients showed statistically significant greater gains in MBI function (mean (SD) baseline 47.31 (28.90) to 55.58 (29.37)) and well-Being (mean (SD) baseline 4.94 (3.95) to 8.46 (3.49)), decreased ill-Being and agitation (mean (SD) baseline 3.04 (2.11) to $0.84(1.26)$ ) and greater improvement in EuroQoL index score (mean (SD) baseline - 0.16 $(0.43)$ to $0.15(0.41))$ after adjusting for baseline differences that translated to a quality-adjusted life years gain of 0.045 , assuming stability over 3 months.

A controlled before-and-after design by Ulin et $a l^{26}$ studied the effect of proactive care-planning based on Gothenburg Person-Centred Care (gPCC). It seeks to identify patient's resources including motivations and goals. This information is used to develop a health plan which includes planned investigations, length of stay in hospital and treatment goals. The health plan is discussed with the patient to reach consensus and the plan is regularly evaluated. The population were recruited from five designated wards at a University Hospital in Sweden. The patients were assessed by a specialised cardiologist before final inclusion, guided by the European Society of Cardiology guidelines for diagnosing congestive heart failure. The study used discharge destination and number of days until the discharge was recorded, to measure the gPCC intervention. They found improved discharge processes (1-5 days for gPCC group vs 1-28 days for control group), and fewer days in hospital (11 days for gPCG group vs 35 days for control group).

An RCT by Willadsen et $a l^{30}$ examined the effect of structured personal diabetes care with general practitioners (GPs) that ask GPs and patient to agree on the best possible goal for controlling risk factors. GPs were offered six seminars and were instructed to give advice lifestyle. Patients were invited to attend follow-up examination quarterly and screening for diabetes complications every year. The study used SRH and diabetes symptoms to measure the effect of the structured person care intervention. They found that the intervention reduced the diabetes symptoms (OR 0.79; 95\% CI 0.64 to 0.97 ), but they did not find the same after 14 years follow-up.
An observational matched cohort study by Wong et $a l^{25}$ implemented a Patient Empowerment Programme (PEP) that aims to provide patient with knowledge and skills about their disease type 2 diabetes mellitus and to facilitate autonomous self-regulation. The programme consisted of generic sessions about self-efficacy enhancement and lifestyle modification as well as disease-specific sessions for a period of up to 12 months. Two nongovernment organisations (NGOs) delivered the intervention, the NGO's invited at general outpatients' clinics or family medicine specialist eligible patients to join the PEP. The study used HbA1c, systolic BP (SBP), diastolic $\mathrm{BP}$ and low-density lipoprotein cholesterol (LDL-C) to measure the effect of PEP. They found improvement in the clinical outcomes. A significantly greater percentage of patients in the PEP group attained $\mathrm{HbA} 1 \mathrm{C} \leq 7 \%$ or LDL-C $\leq 2.6 \mathrm{mmol} / \mathrm{L}$ at 12-month follow-up compared with the non-PEP group. PEP group had a mean 0.813 fewer general outpatient clinic (GOPC) visits in comparison with the non-PEP group.

A study by Hochhalter et $a l^{23}$ measured the effect of Making the Most of Your Healthcare intervention which offered tools and taught skills to (1) prepare for healthcare appointments, (2) communicate effectively and gather information and support during healthcare appointments and (3) follow through on plans of care. The intervention included a 2-hour workshop and two telephone calls individualised to the patient's prehealthcare and posthealthcare appointment needs. The included population were patients in a large Internal Medicine Clinic and had been treated for at least two of seven chronic illnesses. They found a statistically significant improvement in selfefficacy for the intervention group, who received a 2-hour workshop. They used PAM-13 and Health Related Quality of Life-14 questionnaires as measurement. They found an improvement in Self-Efficacy in the Appointment group (mean (SD) baseline 6.9 (1.9) to $7.4(1.8)$ ) mean diff. $0.4795 \%$ CI 0.07 to $0.87, \mathrm{p}=0021$. They did not find any improvement in health for the control group or safety group.

A quasi-experimental study by Mateo-Abad et $a l^{27}$ examined the impact of the CareWell integrated care model on use of health resources and clinical effectiveness. The programme is based on coordination between health providers, patient empowerment and home-based care, supported by communication and information technology tools. Relevant differences were observed between the intervention and control group, including reduced numbers of hospitalisations and visits to emergency centres, and clinical outcomes in the intervention group. For instance, when hospitalised their hospital stay was longer for the control group; the mean number of days in the hospital was 13.3 (SD 13.5), whereas the mean stay for the intervention group was 10.4 (SD 9) days.

Whereas the studies described above did show some improvements of patient-engagement interventions in multimorbid older patients based on a range of outcomes, 
the following three studies did not find any significant improvements in health outcomes.

A pragmatic cluster-randomised trial by Salisbury et $a l^{22}$ examined the effect of the so-called 3D intervention which is based on a patient-centred care model and seeks to improve continuity, coordination and efficiency of care by replacing disease-focused reviews of each health condition with one 6-monthly comprehensive multidisciplinary review. Each 3D review consists of two appointments with a nurse and a named responsible physician and a recordsbased medication review by a pharmacist. The population were recruited from three general practices providing national health service primary medical in England and Scotland. They measured quality of life with a 5Q-5D-5L questionnaire. The intention-to-treat analysis showed no difference between trial groups (adjusted difference in mean EQ-5D-5L 0.00, 95\% CI -0.02 to $0.02 ; p=0.93$ ). They concluded the intervention did not improve the participant's quality of life.

The study by Tinsel $^{20}$ implemented a SDM training programme that aims to enhance the active role of patients. The programme included disease information, physician-patient communication, steps of SDM, motivational interviewing, decision table listing and role plays simulating consultations. The GP's followed a SDM training programme, and the study population was conducted through GP's in southwest Germany. They used change of patients' perceived participation (SDMQ-9) and change in SBP. According to the mixed model analysis, the average change from T0 was 3.11 points higher in the intervention group than in the control group $(97.5 \%$ CI $-2.37 ; 8.61, \mathrm{p}=0.203)$. The effect was not significant at the (Bonferroni-corrected) $2.5 \%$ level. They did not find any statistically significant improvement in SBP.

The study by Schwarze et $a l^{31}$ measured the effect of a question prompt list (QPL) intervention versus usual care among older patients. The QPL intervention target informational needs of patients considering major surgery and include 11 questions that prompt patients and their family members to query their surgeon about treatment options, etc. The study population was conducted among surgeons who perform high-risk oncologic or vascular operations on older patients with comorbidities. They measured patient engagement and well-being, including anxiety in patients. For instance, on average, anxiety scores were 1.3 (95\% CI 0.2 to 2.4 ) points higher for patients in the QPL intervention group. The authors concluded that these effects were less than the minimally important difference and that the QPL intervention in general did not influence patient engagement and wellbeing compared with usual care.

\section{DISCUSSION}

\section{Statement of principal findings}

This systematic review aimed to provide an overview of the effects of patient engagement interventions for older patients with multimorbidity. From the 805 studies identified, only 12 studies met the inclusion criteria. The included studies were heterogeneous in characteristics of populations, number of participants, types of interventions to enhance patient-engagement, length of follow-up and outcome measures. A range of interventions ranging from prompt list to coaching sessions. This diversity in the evidence base challenges the ability to draw robust conclusions. Overall, the majority of studies showed improvements in health and patient-reported outcomes among patients participating in patient engagement interventions. There was some evidence to indicate that the clinical outcomes (BP, Hbalc, diabetes symptoms and glycaemic outcomes) were improved. Furthermore, some evidence indicates improvements in quality of life (EuroQol, Quality-Adjusted Life Years, SRH) and fewer healthcare visits (hospitals, GOPC). However, one study found no significant improvements in quality of life, another study found no significant improvements in patient well-being and anxiety symptoms, and a third study found no significant improvements in BP. As indicated by the limited number of studies and the wide heterogeneity in characteristics of populations, types of interventions to enhance patient-engagement, outcome measures and length of follow-up, there is a need for more substantial studies evaluating patient-engagement tools for both implementation and effect in older patients with multimorbidity using more longer-term outcomes to capture both patient, provider and system-level effects of patientengagement. While our review adds to the important field of ensuring that interventions to enhance patientengagement are developed, implemented and evaluated specifically in the growing population of older adults living with multimorbidity, the review supports previous work in finding too fragile evidence for robust conclusions to be made. ${ }^{10-15}$

\section{Strengths}

This review has several strengths. This review contributes to providing a more substantive evidence base to guide prioritisation and implementation into mainstream healthcare delivery. Since patient-engagement aims at improving care overall, this review did not restrict itself to studies based on particular health outcomes, and consequently studies into a range of health and patientreported outcomes were included.

Another strength is that the systematic literature search that was undertaken adhering to a prespecified protocol. To standardise our assessment process, we used DistillerSR to upload the bibliographic reference information. We performed a wide search to allow different study designs to include methodological heterogeneity. However, the majority of the included studies were RCT. Two researchers independently selected studies collected data and rated quality of included studies using GRADE method. Discrepancies were resolved by consensus. We used a transparent framework for developing and 
presenting summaries of evidence. GRADE is the most widely adopted tool for grading the quality of evidence.

\section{Limitations}

Despite of the systematic approach adopted; this review has its limitations. The literature search was completed using two key databases, but additional peer-reviewed articles might have been found by searching and including from a broader range of sources. Relevant articles were excluded if they were published in languages other than English, Danish, Swedish or Norwegian. Another limitation relates to the differences in transparency as to population characteristics across articles which affected our ability to ascertain types of multimorbid conditions and the extent of multimorbidity in the study populations. A minority of the included studies had small participant numbers which might have affected power of the studies. Two studies included less than 100 patients in total. Metaanalysis was not conducted as there was heterogeneity in the outcomes and measurement tools used in the studies. Overall, the quality of the included studies was of low to moderate. Some aspects of quality assessment and risk of bias were unclear across the included studies. This complicates the overall quality assessment. Furthermore, we did not seek clarification with the study authors about whether our assessment of risk of bias in the individual studies was correct.

\section{Implications}

This review has highlighted the possible improvements in health and patient-reported outcomes among patients exposed to patient-engagement interventions. However, the evidence base is inconsistent and the quality of the studies is relatively low. Further high-quality studies in larger populations over longer time-periods are needed to investigate the long-term effect of patient-engagement interventions.

\section{CONCLUSION}

This systematic review found only limited evidence to support the improvements in health and patient-reported outcomes among older multi-morbid patients exposed to patient-engagement interventions. As the quality of the included studies was mostly low, the findings should be interpreted with caution, and there is a need for more robust studies into efficient approaches to engaging older adults with multimorbidity in care trajectories.

Contributors MBS and KA contributed equally to this paper. MK, MBS and KA designed the study. MBS and KA carried out data collection and analysis. All authors contributed to drafting the manuscript. MK is the guarantor. All authors have read and approved the manuscript.

Funding The authors disclosed receipt of the following financial support for the research, authorship, and/or publication of this article: This work was supported by (Nordea-fonden) grant number (02-2013-0200).

Competing interests None declared.

Patient consent for publication Not applicable.

Provenance and peer review Not commissioned; externally peer reviewed.
Data availability statement Data are available on reasonable request. Data from the literature search and selection process are available on reasonable request to the corresponding author.

Supplemental material This content has been supplied by the author(s). It has not been vetted by BMJ Publishing Group Limited (BMJ) and may not have been peer-reviewed. Any opinions or recommendations discussed are solely those of the author(s) and are not endorsed by BMJ. BMJ disclaims all liability and responsibility arising from any reliance placed on the content. Where the content includes any translated material, BMJ does not warrant the accuracy and reliability of the translations (including but not limited to local regulations, clinical guidelines, terminology, drug names and drug dosages), and is not responsible for any error and/or omissions arising from translation and adaptation or otherwise.

Open access This is an open access article distributed in accordance with the Creative Commons Attribution Non Commercial (CC BY-NC 4.0) license, which permits others to distribute, remix, adapt, build upon this work non-commercially, and license their derivative works on different terms, provided the original work is properly cited, appropriate credit is given, any changes made indicated, and the use is non-commercial. See: http://creativecommons.org/licenses/by-nc/4.0/.

\section{ORCID iDs}

Mathilde Bendix Søgaard http://orcid.org/0000-0002-5262-0530

Katrine Andresen http://orcid.org/0000-0003-0952-9659

Maria Kristiansen http://orcid.org/0000-0003-2319-3637

\section{REFERENCES}

1 American Geriatrics Society Expert Panel on Person-Centered Care. Person-Centered care: a definition and essential elements. J Am Geriatr Soc 2016;64:15-18.

2 Hargraves I, Montori VM, Tilburt JC. Curare Aude-Caring for patients, with them. J Clin Ethics 2020;31:79-82.

3 Pieterse $\mathrm{AH}$, Stiggelbout AM, Montori VM. Shared decision making and the importance of time. JAMA 2019;322:25-6.

4 Johnston MC, Crilly M, Black C, et al. Defining and measuring multimorbidity: a systematic review of systematic reviews. Eur $\mathrm{J}$ Public Health 2019;29:182-9.

5 Davies LE, Spiers G, Kingston A, et al. Adverse outcomes of polypharmacy in older people: systematic review of reviews. J Am Med Dir Assoc 2020;21:181-7.

6 Uijen AA, van de Lisdonk EH. Multimorbidity in primary care: prevalence and trend over the last 20 years. Eur $J$ Gen Pract 2008;14:28-32.

7 van Oostrom SH, Gijsen R, Stirbu I, et al. Time trends in prevalence of chronic diseases and multimorbidity not only due to aging: data from general practices and health surveys. PLoS One 2016;11:e0160264.

8 Spencer-Bonilla G, Thota A, Organick P, et al. Normalization of a conversation tool to promote shared decision making about anticoagulation in patients with atrial fibrillation within a practical randomized trial of its effectiveness: a cross-sectional study. Trials 2020;21:1-10.

9 Fernando AT, Arroll B, Consedine NS. Enhancing compassion in general practice: it's not all about the doctor. Br J Gen Pract 2016;66:340-1.

10 Sepucha KR, Simmons LH, Barry MJ, et al. Ten years, forty decision aids, and thousands of patient uses: shared decision making at Massachusetts General Hospital. Health Aff 2016;35:630-6.

11 Rashid A. Choosing together: encouraging person centred care and shared decision making. BMJ 2015;350:h2935.

12 Barry MJ, Edgman-Levitan S. Shared decision making - the pinnacle of patient-centered care. $N$ Engl J Med Overseas Ed 2012;366:780-1.

13 Plsek P. Crossing the quality chasm: a new health system for the 21 st century: the National academies of medicine 2001.

14 Montori VM, Kunneman M, Brito JP. Shared decision making and improving health care: the answer is not in. JAMA 2017;318:617-8.

15 Butterworth JE, Hays R, McDonagh STJ, et al. Involving older people with multimorbidity in decision-making about their primary healthcare: a Cochrane systematic review of interventions (abridged). Patient Educ Couns 2020;103:2078-94.

16 Elwyn G, Frosch DL, Kobrin S. Implementing shared decisionmaking: consider all the consequences. Implementation Sci 2015;11:114.

17 Guyatt G, Oxman AD, Akl EA, et al. Grade guidelines: 1. IntroductionGRADE evidence profiles and summary of findings tables. J Clin Epidemiol 2011;64:383-94. 
18 Sterne JA, Hernán MA, Reeves BC, et al. ROBINS-I: a tool for assessing risk of bias in non-randomised studies of interventions. BMJ 2016;355:i4919.

19 Higgins JPT, Altman DG, Gøtzsche PC, et al. The Cochrane collaboration's tool for assessing risk of bias in randomised trials. BMJ 2011;343:d5928.

20 Tinsel I, Buchholz A, Vach W, et al. Shared decision-making in antihypertensive therapy: a cluster randomised controlled trial. BMC Fam Pract 2013;14:135.

21 Shively MJ, Gardetto NJ, Kodiath MF, et al. Effect of patient activation on self-management in patients with heart failure. $J$ Cardiovasc Nurs 2013;28:20-34.

22 Salisbury C, Man M-S, Bower P, et al. Management of multimorbidity using a patient-centred care model: a pragmatic cluster-randomised trial of the 3D approach. Lancet 2018;392:41-50.

23 Hochhalter AK, Song J, Rush J, et al. Making the most of your healthcare intervention for older adults with multiple chronic illnesses. Patient Educ Couns 2010;81:207-13.

24 Tay FHE, Thompson CL, Nieh CM, et al. Person-centered care for older people with dementia in the acute Hospital. Alzheimers Dement 2018;4:19-27.

25 Wong CKH, Wong WCW, Lam CLK, et al. Effects of patient Empowerment programme (PEP) on clinical outcomes and health service utilization in type 2 diabetes mellitus in primary care: an observational matched cohort study. PLoS One 2014:9:e95328.

26 Ulin K, Olsson L-E, Wolf A, et al. Person-centred care-An approach that improves the discharge process. Eur $J$ Cardiovasc Nurs 2016;15:e19-26.

27 Mateo-Abad M, González N, Fullaondo A, et al. Impact of the CareWell integrated care model for older patients with multimorbidity: a quasi-experimental controlled study in the Basque country. BMC Health Serv Res 2020;20:613.

28 Naik AD, Hundt NE, Vaughan EM, et al. Effect of Telephone-Delivered collaborative goal setting and behavioral activation vs enhanced usual care for depression among adults with uncontrolled diabetes: a randomized clinical trial. JAMA Netw Open 2019;2:e198634.

29 Reed RL, Roeger L, Howard S, et al. A self-management support program for older Australians with multiple chronic conditions: a randomised controlled trial. Med J Aust 2018;208:69-74.

30 Willadsen TG, Siersma V, Nielsen ABS, et al. The effect of structured personal care on diabetes symptoms and self-rated health over 14 years after diabetes diagnosis. Prim Care Diabetes 2018;12:354-63.

31 Schwarze ML, Buffington A, Tucholka JL, et al. Effectiveness of a question prompt list intervention for older patients considering major surgery: a multisite randomized clinical trial. JAMA Surg 2020;155:6-13. 